\title{
Mesenteric panniculitis: can venous anatomy and malignancy be a trigger cause?
}

\author{
Mehmet Ali Gültekin
}

Department of Radiology, Bezmialem Vaklf University School of Medicine, Istanbul, Turkey

\section{ABSTRACT}

Objectives: To investigate whether portal vein (PV), splenic vein (SV), superior mesenteric vein (SMV) diameters and portomesenteric angle (PMA) may play a role in the etiology of mesenteric panniculitis (MP). To investigate relationship between MP and malignancy.

Methods: We included 70 patients with MP and 70 patients age, gender, sagittal abdominal diameter randomly matched control group, retrospectively.

Results: We found slightly higher PV and SMV diameter and slightly lower SV diameter in MP patients compared with control group. But these results were not statistically significant $\left(p_{1}=0.321\right.$ and $p_{2}=0.147, p_{1}$ $=0.540$ and $p_{2}=0.185, p_{1}=0.216$ and $p_{2}=0.617$, for two observers respectively). We found higher PMA in MP patients compared with control group but this difference is not statistically significant $\left(p_{1}=0.252\right.$ and $p_{2}$ $=0.366$, for two observers). Twenty three $(32.9 \%)$ of 70 patients diagnosed MP have underlying malignancy while $17(24.3 \%)$ of 70 control subject were coexisted malignancy. But this difference is not statistically significant $(p=0.262)$. Interobserver agreement was excellent in terms of SMV and SV diameters and PMA measurements (ICC were $0.927,0.911$ and 0.965 respectively), good for PV diameters (ICC was 0.884 ).

Conclusions: Study results show that MP is not associated with PV, SMV and PMA. Contrary to some studies, there is no relationship between MP and malignancy.

Keywords: Mesenteric panniculitis, computed tomography, portomesenteric angle, malignancy

$\mathbf{M}$ esenteric panniculitis (MP) is an uncommon disease characterised by chronic nonspecific inflammatory process that affects the adipose tissue of the bowel mesentery [1]. MP has several synonyms such as retractile mesenteritis, mesenteric lipodystrophy and sclerosing mesenteritis $[2,3]$. MP mostly occurs in mid to late adulthood, showing a male predominance and with a prevelence of $0.16 \%-2.5 \%$ $[1,4]$. Histopathologically, altered adipocytes and lipid laden macrophages with mild inflammatory reaction and fibrosis were showed [5]. MP has various clinical presentations, up to a third of patients may be symptomatic [6]. MP is most frequently diagnosed incidentally by computed tomography (CT), but it also can be diagnosed with magnetic resonance imaging and abdominal sonography [6].

Although etiology of the disease is unclear, variety of conditions such as vasculitis, granulomatous disease, rheumatic disease, malignancies, trauma, pancreatitis, autoimmune disorders, ischemia and pre $\neg$ vious abdominal surgery are thought to be related to the disease. Especially relationship with malignancy 
has been extensively studied in the literature and conflicting results were reported [1-7].

It is known that prolonged venous congestion causes edema, cell atrophy, necrosis and with time fibrosis develops. Chronic venous congestion and increased hydrostatic pressure may cause venous dilatation. According to this finding we hypothesized that MP may develop due to chronic congestion and this may be associated with increased portal vein (PV), splenic vein (SV) and superior mesenteric vein (SMV) diameters. Similarly, portomesenteric angle (PMA) may also affect blood flow and venous return and may be associated with increased hydrostatic pressure and venous congestion.

This study has 3 main objectives. First, to find if there is any correlation between PV, SMV, SV diameters and MP. Second, to investigate the association between PMA and MP. And third, to determine the presence and rate of malignancy accompanying MP.

\section{METHODS}

\section{Patients}

We retrospectively examined 100 patients with diagnosis of MP who underwent $\mathrm{CT}$ examination in our radiology department between January 2017 and March 2020. Diagnostic criteria for the MP were described as 1) presence of a well-defined fatty mass at the root of the mesentery displacing neighboring structures 2) a higher attenuation and inhomogeneity than retroperitoneal, mesocolon or subcutaneous fat tissue; 3) containing small lymph nodes within the fatty mass; 4) a hypodense fatty halo sign that surrounding vessels and lymph nodes and 5) a hyper-attenuating pseudocapsule surrounding the mesenteric fatty mass [6]. Presence of at least 3 of the 5 typical signs required for diagnosis. All CT images were re-evaluated and diagnosis was confirmed by two abdominal radiologist seperately (MAG and RS).

Exclusion criteria were determined as lack of IV contrast administration $(n=22)$, presence of a tumor invading the portal vein that may affect the venous diameters $(n=4)$, advanced stage cirrhosis $(n=1)$, heavy image artefacts and technical reasons $(n=3)$ (Fig. 1). A total of 70 patients with diagnosis of MP included the study. For each patient with MP, one control patient without evidence of MP was selected. Con- trol groups were matched for age, gender, sagittal abdominal diameter (SAD) and CT protocol. Our institutional ethical committee approved the study protocol (decision number: 08/147, date:11.06.2020). Details of the identified clinical data are shown in Table 1.

\section{Imaging Protocol}

All CT images obtained using a 16-section multi detector CT system (Aquilion 16; Toshiba Medical Systems, Japan). Abdominopelvic CT was performed in the craniocaudal direction from the level above the diaphragm to the symphysis pubis. The patients were instructed to hold their breath with tidal inspiration during examination. CT parameters were as follows: $32 \times 1 \mathrm{~mm}$ collimation, 1.25 pitch, 0.5 seconds rotation time, $5 \mathrm{~mm}$ reconstructed section thickness and $5 \mathrm{~mm}$ intersection gap, $120 \mathrm{kV}$ tube voltage, $250 \mathrm{~mA}$ tube current-time product. The axial section data were reconstructed at a thickness of $5 \mathrm{~mm}$ with $5-\mathrm{mm}$ increments and a thickness of $2 \mathrm{~mm}$ with 1-mm increments. Maximum intensity projection (MIP) images were reconstructed by using coronal reformatted images with

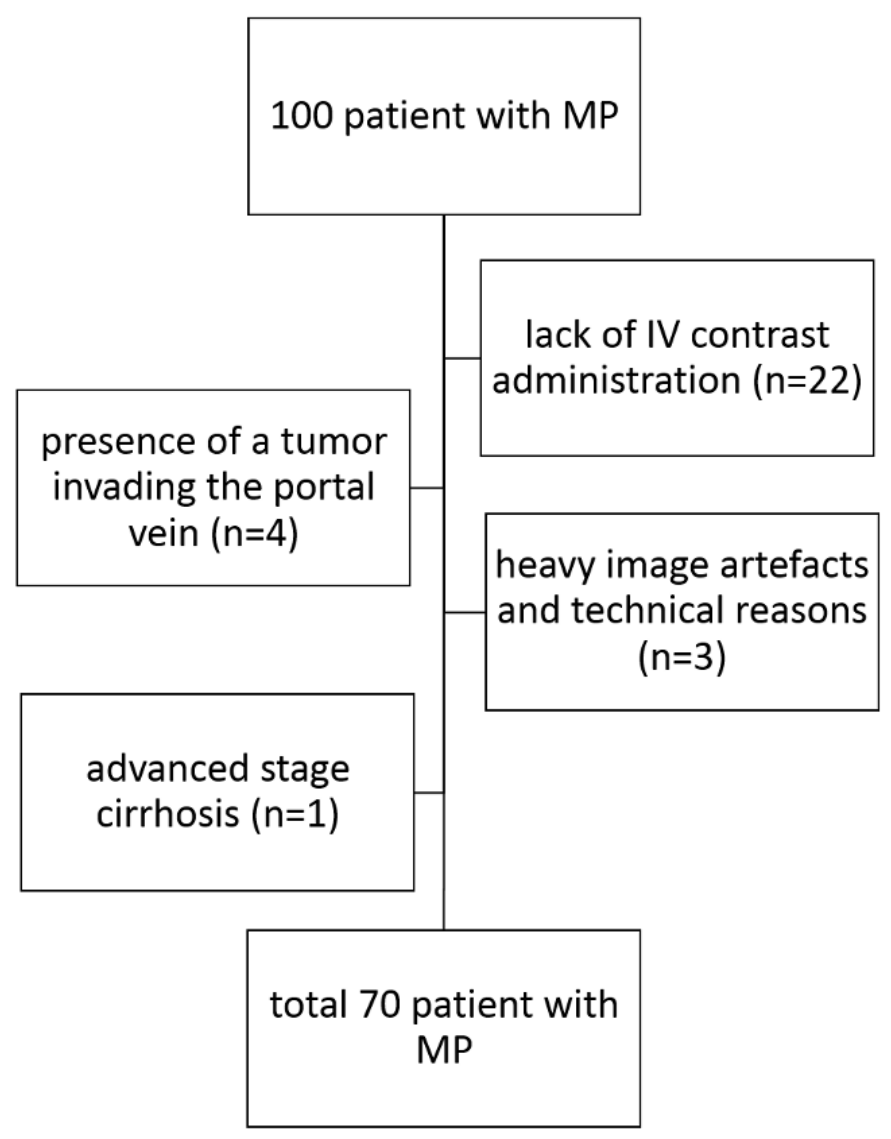

Fig. 1. Diagram showing the exclusion criteria of patients for study. MP = mesenteric panniculitis 
Table 1. Baseline patient characteristics

\begin{tabular}{lccc}
\hline & $\begin{array}{c}\text { MP patients } \\
(\mathbf{n}=\mathbf{7 0})\end{array}$ & $\begin{array}{c}\text { Control group } \\
(\mathbf{n}=\mathbf{7 0})\end{array}$ & $\boldsymbol{p}$ value \\
\hline Age, years & $57.7 \pm 10.9$ & $57.4 \pm 13.1$ & 0.905 \\
Gender (female/male) & $33 / 37$ & $33 / 37$ & 1 \\
SAD, $\mathrm{mm}$ & $255 \pm 21.6$ & $254.9 \pm 31.6$ & 0.988 \\
$($ mean \pm SD) & & & \\
\hline
\end{tabular}

$\mathrm{MP}=$ mesenteric panniculitis, $\mathrm{SAD}=$ sagittal abdominal diameter, $\mathrm{SD}=$ standart deviation

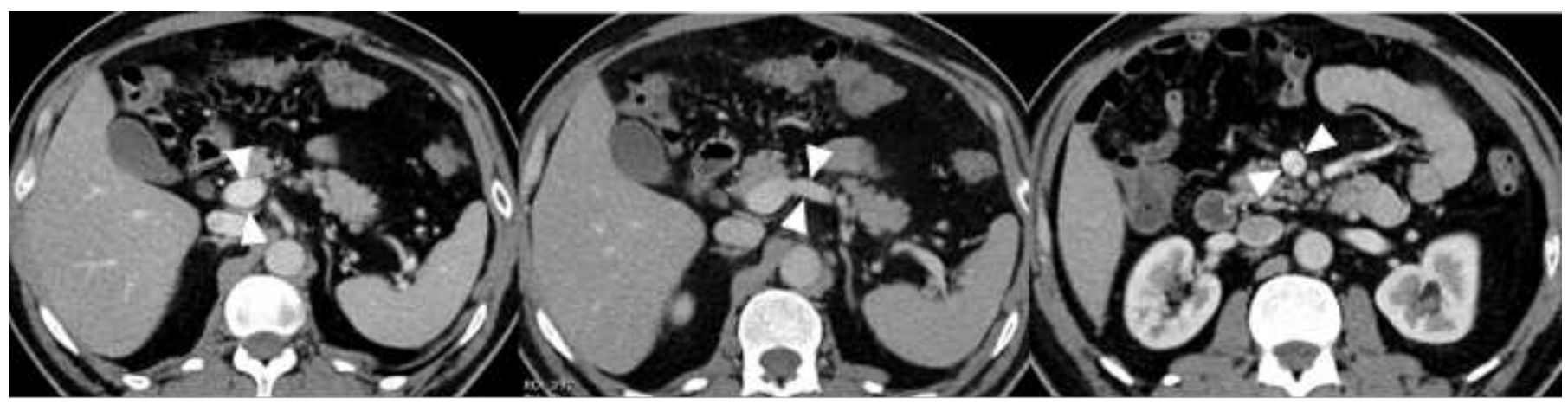

Fig. 2. Contrast enhanced axial CT image shows measurements for portal vein, splenic vein and superior mesenteric vein (arrowheads).

64 slices on the our PACS system (FUJIFILM Medical Systems USA). Seventy milligrams of intravenous contrast material (iohexol; Omnipaque 350, GE Healthcare, Cork, Ireland) was administered in all patients at a rate of $3 \mathrm{ml} / \mathrm{s}$ with a power injector. CT was started 70 seconds after start of contrast material injection.

\section{CT Measurements}

PV, SMV and SV diameters were measured outer wall-to-outer wall on axial images on the portal venous phase (Fig. 2). All measurements were performed at the point of $1 \mathrm{~cm}$ distal (SMV and splenic vein) and proximal (portal vein) to the portal confluence. MIP images were used for PMA measurements. A line parallel to the main portal vein was drawn from central section of the portal vein at the branching level. A second line was drawn from the central point of the portal confluence to the distal cental point of mesenteric vein at the branching level. Angle between the two lines was measured and recorded (Fig. 3). All images were evaluated by two abdominal radiologist having more than 5 years of experience in abdominal imaging (MAG and RS).

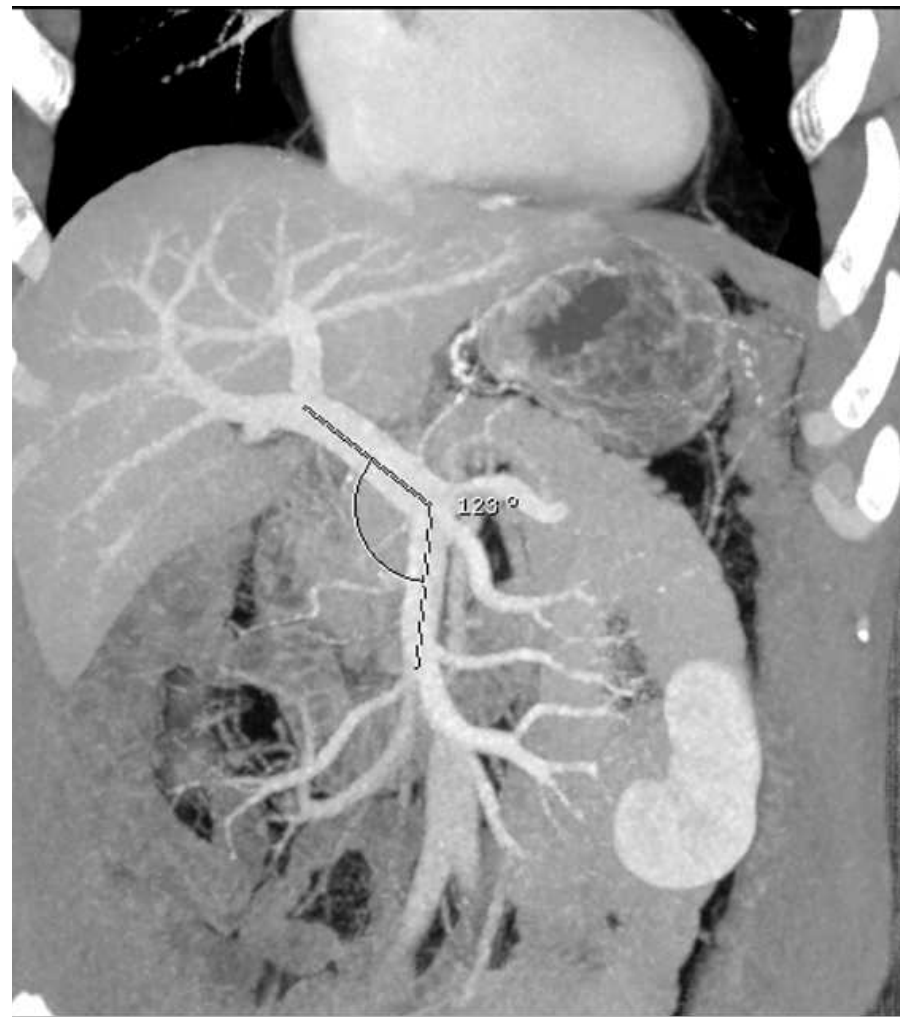

Fig. 3. Contrast enhanced, coronal CT maximum intensity projection image shows measurement of portomesenteric angle. 


\section{Statistical Analysis}

Statistical analysis was performed by the IBM SPSS Statistics 20.0 statistical software (Armonk, NY: IBM Corp.). Kolmogorov-Smirnov test was used for normality. Mean \pm standard deviations presented as descriptive statistics. In the comparisons of the PV, SMV, SV diameters and PMA in the MP and control group the independent samples t-test was used. Pearson Chi-Squared test was used to determine the presence of concomitant malignancy for two groups. A $p$ value below 0.05 was accepted as statistical significance. Interobserver agreement for evaluation of all measurements was performed. The intraclass correlation coefficient (ICC) was calculated to assess the degree of agreement between two observers for PV, SMV and SV diameters and PMA measurements.

\section{RESULTS}

A total of 70 patients with diagnosed MP (33 females, 47.1\%: 37 males, 52.9\%) and age, gender and abdominal diameter matched 70 control group (33 females, $47.1 \%$ : 37 females, 52.9\%) were included the study. The mean age of the samples were $57.7 \pm 10.8$ years and $57.4 \pm 13.1$ years, for MP and control group, respectively. The SADs of the samples were $255 \pm$ $21.6 \mathrm{~mm}$ and $254.9 \pm 31.6 \mathrm{~mm}$, for MP and control group respectively. As populations were matched, there was no difference in gender, age and SAD between patients and control group.

There were no statistically significant differences between MP and control group in terms of PV diameter $\left(p_{1}=0.321\right.$ and $p_{2}=0.147$, for two observers), SMV diameter $\left(p_{1}=0.540\right.$ and $p_{2}=0.185$, for two observers), SV diameter $\left(p_{1}=0.216\right.$ and $p_{2}=0.617$, for two observers) and PMA ( $p_{1}=0.252$ and $p_{2}=0.366$, for two observers). These results are shown in Table 2.

Twenty three (32.9\%) of 70 patients diagnosed MP have underlying malignancy while 17 (24.3\%) of 70 control subject were coexisted malignancy. But this difference is not statistically significant $(p=0.262)$. These results are summarized in Table 2.

Underlying malignancies with MP were colorectal $(\mathrm{n}=7)$, breast $(\mathrm{n}=3)$, lymphoma $(\mathrm{n}=2)$, gastric $(\mathrm{n}=$ $2)$, renal $(n=2)$, prostatic $(n=1)$, cervical $(n=1)$, endometrial $(n=1)$, pancreas $(n=1)$, lung $(n=1)$, bladder $(n=1)$ and testis $(n=1)$. Remaining 47 of 70 $(67.1 \%)$ patient diagnosed MP had no underlying malignancy.

Interobserver agreement was excellent in terms of

Table 2. Comparison of PV, SV, SMV diameter, PMA and concomitant malignancy rates of two group

\begin{tabular}{|c|c|c|c|c|c|c|c|}
\hline & \multicolumn{2}{|c|}{$\begin{array}{l}\text { MP patients } \\
\quad(n=70)\end{array}$} & \multicolumn{2}{|c|}{$\begin{array}{c}\text { Control group } \\
(n=70)\end{array}$} & \multicolumn{2}{|c|}{$p$ value } & \multirow[t]{2}{*}{ ICC } \\
\hline & $\begin{array}{c}\text { Observer } \\
1\end{array}$ & $\begin{array}{c}\text { Observer } \\
2\end{array}$ & $\begin{array}{c}\text { Observer } \\
1\end{array}$ & $\begin{array}{c}\text { Observer } \\
2\end{array}$ & $\begin{array}{c}\text { Observer } \\
1\end{array}$ & $\begin{array}{c}\text { Observer } \\
2\end{array}$ & \\
\hline $\begin{array}{l}\text { PV diameter, mm } \\
(\text { mean } \pm \mathrm{SD})\end{array}$ & $140.6 \pm 17.9$ & $139.6 \pm 16.9$ & $137.8 \pm 14.1$ & $135.6 \pm 15.6$ & 0.321 & 0.147 & 0.884 \\
\hline $\begin{array}{l}\text { SV diameter, mm } \\
(\text { mean } \pm \mathrm{SD})\end{array}$ & $88.7 \pm 14.5$ & $89.3 \pm 13.7$ & $91.9 \pm 15.4$ & $90.6 \pm 16.8$ & 0.216 & 0.617 & 0.911 \\
\hline $\begin{array}{l}\text { SMV diameter, } \\
\mathrm{mm} \\
(\mathrm{mean} \pm \mathrm{SD})\end{array}$ & $121.5 \pm 17.5$ & $120.6 \pm 17.4$ & $116.2 \pm 14.3$ & $116.9 \pm 15.4$ & 0.540 & 0.185 & 0.927 \\
\hline $\begin{array}{l}\text { PMA, degree } \\
(\text { mean } \pm \text { SD) }\end{array}$ & $131.1 \pm 12.6$ & $130.9 \pm 13.5$ & $128.2 \pm 17.3$ & $128.4 \pm 18.5$ & 0.252 & 0.366 & 0.965 \\
\hline $\begin{array}{l}\text { Concomitant } \\
\text { malignancy, n (\%) }\end{array}$ & \multicolumn{2}{|c|}{$23(32.9 \%)$} & \multicolumn{2}{|c|}{$17(24.3 \%)$} & \multicolumn{2}{|c|}{0.262} & \\
\hline
\end{tabular}

$\mathrm{ICC}=$ intraclass correlation coefficient, $\mathrm{MP}=$ mesenteric panniculitis, $\mathrm{SD}=$ standart deviation, $\mathrm{PMA}=$ portomeseneric angle, $\mathrm{PV}=$ portal vein, $\mathrm{SMV}=$ superior mesenteric vein, $\mathrm{SV}=$ splenic vein 
lesion SMV and SV diameters and PMA measurements (ICC were $0.927,0.911$ and 0.965 respectively), good for PV diameters (ICC was 0.884).

\section{DISCUSSION}

MP refers to $\mathrm{CT}$ appearance of increased density of the intestinal mesentery by chronic nonspecific inflammation and fibrosis. Although the exact etiology is still unknown, various mechanisms underlying MP have been discussed in the literature such as previous abdominal surgery, trauma, autoimmunity, ischemic injury and paraneoplastic syndrome [7]. Especially its relationship with malignancy has been extensively studied and there are contradictory results in the literature. While some studies showed increased risk of malignancy [1, 3, 8-10], some studies have reported contrary results $[6,7,11]$. In the current study, $32.9 \%$ of patients diagnosed with MP had underlying malignancy while $24.3 \%$ of control subjects had underlying malignancy. Although higher rate of malignancies have been found in MP patients, this difference is not statistically significant $(p=0.262)$. Pathophysiological process between mesenteric panniculitis and the development of malignancy is also unknown. Kipfer et al. [5] suggested that mesenteric panniculitis is a nonspecific response to an underlying abdominal malignancy. However, it was reported that extraabdominal malignancies can also accompany MP [1]. Four of 23 patients had extraabdominal malignancy ( 3 breast carcinoma and 1 lung carcinoma) in our study.

Conditions that may affect the vascular supply of the mesentery such as abdominal surgery or mesenteric thrombosis were also associated with mesenteric panniculitis $[12,13]$. Seo et al. [3] found dilated vessels within the misty mesentery in 27 (93\%) of the 29 patients with MP. More of them had venous dilatation (23 of 27 patients). They were in the opinion that venous dilatation resulted from hydrostatic pressure or from vascular compression of the vein draining the mesentery [3]. In the lights of these findings we thought that there may be a correlation between PV, SV, SMV diameters and MP. PMA may also affect the venous return that may causes increased hydrostatic pressure and congestion. We found slightly higher PV and SMV diameter in MP patients compared with control group. However these differences are not statisti- cally significant. On the contrary, SV diameters were slightly lower in MP patients compared with control group. Additionally, we found slightly higher PMA in MP patients compared with control group but its not significant. Especially, dilated SMV, that draining the mesentery, may be associated with increased congestion and fibrosis. Higher PMA may cause decreased venous return and increased hydrostatic pressure, congestion and fibrosis.

Our study has some limitations. First one is the retrospective design of the study. Second, lack of histopathologic confirmation of the MP. Diagnosis of MP was made based on diagnostic CT criteria. None of our patients underwent histopathological examination because histopathological examination is unnecessary in most cases. Third, MP and control groups were consisted of selected patients with known diagnosis. This may cause bias in measurements.

\section{CONCLUSION}

As to our knowledge this is the first study to investigate venous diameter in MP. Our study results showed slightly increased PV, SMV diameter and PMA in MP however it is not significant with exellent and good interobserver agreement. We also investigated association between malignancy and MP. Although higher rate of malignancies have been found in MP patients, contrary to some studies this difference is not statistically significant. With these results, we think that venous anatomy and precense of malignancy are not included in the etiology of MP. Larger studies are needed to prove the exact etiology of MP.

\section{Authors' Contribution}

Study Conception: MAG; Study Design: MAG; Supervision: MAG; Funding: MAG; Materials: MAG; Data Collection and/or Processing: MAG; Statistical Analysis and/or Data Interpretation: MAG; Literature Review: MAG; Manuscript Preparation: MAG and Critical Review: MAG.

\section{Conflict of interest}

The authors disclosed no conflict of interest during the preparation or publication of this manuscript.

\section{Financing}


The authors disclosed that they did not receive any grant during conduction or writing of this study.

\section{REFERENCES}

1. Daskalogiannaki M, Voloudaki A, Prassopoulos P, Magkanas E, Stefanaki K, Apostolaki E et al. CT evaluation of mesenteric panniculitis: prevalence and associated diseases. AJR Am J Roentgenol 2000;174:427-31.

2. Sharma P, Yadav S, Needham CM, Feuerstadt P. Sclerosing mesenteritis: a systematic review of 192 cases. Clin J Gastroenterol 2000;10:103-111.

3. Seo BK, Ha HK, Kim AY, Kim TK, Kim MJ, Byun JH et al. Segmental misty mesentery: Analysis of CT features and primary causes. Radiology 2003;226:86-94.

4. Sahin A, Artas H, Eroglu Y, Tunc N, Demirel U, Bahcecioglu IH et al. An overlooked potentially treatable disorder: idiopathic mesenteric panniculitis. Med Princ Pract 2017;26:567-72.

5. Kipfer RE, Moertel CG, Dahlin DC. Mesenteric lipodystrophy. Ann Intern Med 1974;80:582-8.

6. Gögebakan Ö, Albrecht T, Osterhoff MA, Reimann A. Is mesenteric panniculitis truely a paraneoplastic phenomenon? A matched pair analysis. Eur J Radiol 2013;82:1853-9.
7. Protin-Catteau L, Thiefin G, Barbe C, Jolly D, Soyer P, Hoeffel C. Mesenteric panniculitis: review of consecutive abdominal MDCT examinations with a matched-pair analysis. Acta Radiol 2016;57:1438-44.

8. Coulier B. Mesenteric panniculitis. Part 2: prevalence and natural course: MDCTprospective study. JBR-BTR 2011;94:241-6.

9. Canyigit M, Koksal A, Akgoz A, Kara T, Sarisahin M, Akhan

O. Multidetector-row computed tomography findings of sclerosing mesenteritis with associateddiseases and its prevalence. Jpn J Radiol 2011;29:495-502.

10. Nakatani K, Nakamoto Y, Togashi K. FDG-PET/CT assessment of misty mesentery: feasibility for distinguishing viable mesenteric malignancy from stable conditions. Eur J Radiol 2013;82:e380-5.

11. Halligan S, Plumb A, Taylor S. Mesenteric panniculitis: systematic review of crosssectional imaging findings and risk of subsequent malignancy. Eur Radiol 2016;26:4531-7.

12. Soumerai S, Kirkland WG, McDonnell WV, Schanz A. Nodular mesenteritis: report of a case simulating carcinoma of the sigmoid colon and analysis of its histologic profile. Dis Colon Rectum 1976;19:448-52.

13. Pemberton J, Comfort MW, Fair E, Zaslow B. Intestinal lipodystrophy (Whipple's disease). Surg Gynecol Obstet 1947;85:85-91. 